\title{
A Survey on Traffic Sign Detection Techniques Using Text Mining
}

\author{
S. Murugan ${ }^{1}$ and R. Karthika ${ }^{2}$ \\ ${ }^{1}$ Associate Professor, ${ }^{2}$ Research Scholar \\ ${ }^{1 \& 2}$ Department of Computer Science, Nehru Memorial College, Trichy, Tamil Nadu, India \\ E-Mail: Murugan_nmc@hotmail.com, tkarthika_09cs@yahoo.com
}

\begin{abstract}
Traffic Sign Detection and Recognition (TSDR) technique is a critical step for ensuring vehicle safety. This paper provides a comprehensive survey on traffic sign detection and recognition system based on image and video data. The main focus is to present the current trends and challenges in the field of developing an efficient TSDR system. The ultimate aim of this survey is to analyze the various techniques for detecting traffic signs in real time applications. Image processing is a prominent research area, where multiple technologies are associated to convert an image into digital form and perform some functions on it, in order to get an enhanced image or to extract some useful information from it. Keywords: Traffic Sign, Detection, Recognition, Image Processing
\end{abstract}

\section{INTRODUCTION}

Traffic signs are boards and markers placed along with roadsides to convey information to the road users. But, there is a huge variation in the process of detection, segmentation and extraction of information from the sign board. Detection of such signs while moving, interpreting andconveying to the road users is complicated one.

The traffic sign detection problem is broadly classified into two symbols. One is Symbol Based Traffic Sign detection and Text Based Traffic Sign Detection. Further, considering the symbol based detection model is classified into Color based model, Shape based detection model and sliding window based model. The first part of this survey focuses on the symbol based traffic sign detection models. Symbols include the various traffic signs which are used to detect traffic sign clearly.

\section{COLOR-BASED METHODS}

Color-based methods usually use normalized color spaces with a designed threshold to extract the region of interest, where color is clustered by categorizing the pixels with similar or same colors.Then forming a candidate region of interest, next such candidate regions formed are analyzed and the Connected Components (CC)isestimated. The major obstacle here is the degree of clustering done, if found to be over clustered. The scene background in text region may overlap causing confusion, if the data is under clustered. The number of clustering which in turn increases the overheadand cause the system performance to degrade.
Timofte et al., [1] proposed a model which combines 2D and 3D analysis based on RGB channels. The candidate extraction model which is very fast preprocessing step, where an optimized combination of simple (i.e. computationally cheap) and adjustable extraction methods selects bounding boxes. Next, the extracted candidates are verified by a binary classifier which filters out remaining background regions. Recognition - Six one-against-all Support Vector Machine (SVM) classifiers select one of the six basic traffic sign subclasses (triangleup, triangle-down, circle-blue, circle-red, rectangle and diamond) for the different candidate traffic signs. This model works on the Red Green Blue (RGB) color channels which normalized by the intensity variance of each of the colors

Escalera et al., [2] developed a genetic algorithm for detection step and in addition to recognizing the sign the system provides a novel model of information about its state - good, bad or worse, wherein the algorithm, not only knows the detected sign, but, also the confidence in its detection and is believed to help recognition algorithm in a continuous mode when the same sign will appear in several images.

Miura et al., [3] examined an active vision model which both detects and recognizes traffic signs on-line in real time traffic.It has been tested under various lighting conditions by using the multiple thresholds binarization in conjunction with screening by edges for robust detection of the traffic signs.

Gonzalez et al., [4] applied color segmentation to detect the candidate regions. The classification is done using SVM or Naïve Bayes. Gonzalez et al., [5] made use of MSERs for the detection of both traffic signs and text characters. They applied a method based on color segmentation and the BOVW algorithm was applied on each frame to detect white and blue traffic panels. Extracted local key descriptors at some key-points (regions) of interest after applying blue and white color segmentation to the scenes, after which the images were represented as a "bag of visual words" to form a set. This visual region was then classified using SVM and NB Naive Bayes classifiers to the 10763 images taken from Google Street View database.

The second most widely used model is the shape model under the symbols category shapeis another important clue to distinguish the traffic signs withother objects, since the 
shape of these traffic signs is relativelyfixed such as circle, triangle, and square.

Ruta et al., [6] explored the circular Hough transform with an appropriate post-processing novel adaboost algorithm to learn the traffic sign similarity measure from submitted sample image pairs labeled either "same" or "different" to have an either or clause, which enables the construction of an efficient multi-class classifier. The classifier built is robust, fast and efficient tracker that effectively corrects the affine distortions the road signs are normally subjected to and despite this provide a in vehicle detection recognition model.Garcia et al., [7] explored a model which using the Hough transforms detected from the information of the edges of the image. Second, classification, using a neural network, and third, tracking, making use of a Kalman filter, which provides the system with memory where 30 milliseconds per frame is the normal processing time making it applicable in real time situations.

Barnes et al., [8] developed an adaptive model where realtime sign detection was done to reduce the number of pixels.Sign detection on the image using computationally required less time expensive and less overheads algorithms. They used a fast radial symmetry detector stream pictures from a camera mounted inside a car which almost eliminated all non-sign pixels from the input video. Next, they were able to apply normalised cross-correlation to classify the signs. But, the biggest drawback was that the method is suitable for circular signs only.

Barneset al., [9] presented another radial symmetry detectorwhich was used for detecting speed signs. The model has a built in classification series of sequences from roads around Canberra in Australia. Experiments demonstrated running online in the car to be highly effective for sign detection as well as for recognition and has got low false-negativesand low false-positives. The model is a fast radial symmetry detector runs in real time and being a pixelbased shape detector is robust under changing lighting conditions that cause problems for color-based detectors.

Loy et al., [10]created a new method for detecting triangular, square and octagonal road signs by using the symmetric nature of such shapes along with the edge orientations. It exploited the fact that equiangular polygons havea known number of sides and to find possible shape centroid locations is easy in the captured picture. Hence they received a shape invariant model and got the location. The model has a very high accuracy on still images with a detection rate of nearly 95\%. Some shape based models use Hough Transform and its variant which are commonly used to localize the various signswiththe general object detection while a sliding window with a regionclassifieris adopted to assess whether the currentwindow is the traffic sign.

Hanwell et al., [11] examined a system for detecting and tracking the lanes of roads. The drivers are warned well in advance when they change their lanes. The model uses a Hough transformation system, which detects and finds the road marking which are linear and the ones with common vanishing point. They also used an extended version of the Kalman filter to detect and track lanes for the warnings to the drivers.

Greenhalgh et al., [12] introduced a novel system for automatic detection and recognition of the traffic signs where the candidate regions are detected using maximum stable external regions called popularly as MSER. In MSER model, SVM classifiers are used for recognizing the various traffic signs which are trained using histograms of oriented gradient features and this approach is used for detection of all types of traffic signs.

Fischler et al., [13] proposed a new model called Random Sample Consensus (RANSAC)a fitting model which is capable of smoothing data containing errors called hypothetical inliners, The nearest match that fits these models are called the consensus data set and it produces good results.Sliding Window models come at last under the symbols based detection model wherein the detection sign boards fall into a sliding window.

Kwangyong Lim et al., [14] recognized a traffic sign based on general purpose GPU and deep-learningused parallel processing techniques implemented on a GPGPU and thereby considerably reduces the detection time when compared with the sliding-window model. The model uses a cascade classifier that assembles the SVMto verify the candidate region and a CNN for diverse traffic sign recognition.

Creusen et al., [15] converted the image input pixels into HOG features. Object detection is performed by sliding a detection window over the image, where the problem of scale arises and to overcome the preprocessing and detection process is repeated for downscaled versions of the input video frames. The experimental results prove that the generic HOG algorithm significantly outperforms the dedicated algorithm in by over 10-30\% significantly.

Tao Chen et al., [16]boosted decision trees can be used as the sliding window input to detect a test image and generate a set of sign candidate rectangles, which contain both true positives and false positives. Accurate and Efficient Traffic Sign Detection Using Discriminative AdaBoostand SVM to detect as many ground-truth traffic signs as possible.Fine tuning the parameters of the feature pyramid method, e.g., the pyramid level numbers, the sliding window size and length/width ratios, and the boosted decision tree numbers to produce high recall

\section{SLIDING-WINDOW METHODS}

Ivo Creusen et al., [17] proposed the sliding window object detection algorithm which is employed for traffic sign detection with less computational overheads. Sliding- 
window object detectors often use a linear SVM kernel to classify the features in a window that slides across.

Dongdong Wang et al., [18]proposed a fast traffic sign detection model based on a cascade method with saliency test and neighboring scale awareness, wherein the feature maps of several channels are extracted by approximation techniques by the use of sliding windows which are pruned hierarchically using coarse-to-fine classifiers and the correlation between neighboring scales. The cascade model has only one parameter, whereas multiple thresholds are selected to increase speed and also uses a novel saliency test to pre-prune background windows images. The models experiments on two public traffic sign data sets achievea performance which is nearly 7 times faster than existing methods.

The second broad classification is Text Based Detection models. Approaches to text based problems can be broadly divided into two groups: region based methods, and Connected Component (CC) based methods. While regionbased text detection methods use local features like as texture to locate text regions, the connected components CC-based methods attempt to segment text characters individually by using additional information like intensity, color distribution, and edges across the image. They normally consist of three phases: a first stage to detect CCs within the image, a second stage to eliminate unlikely CCs based on their features, and a final stage that attempts to group the remaining CCs into words or lines.

\section{TEXTURE BASED METHODS}

Li et al. [19] detected and tracked the texts in videos, where images are decomposed by hybridwavelets and each window is classified as text or non-text by a neural network. Chen et al., [20] proposed a fast text detector by using a strong AdaBoost classifier trained on a set of specified features. Wang et al., [21] first use sliding window to detect the charactersand then calculate the scores of all possible combinations of characters according to the location or positioninformation and at last, the most likely words are selected from a given word list. But, these methods suffer from computational complexity.

\section{COMPONENT-BASED METHODS}

Epshtein et al., [21] proposed a text detection algorithm: Stroke Width Transform (SWT), makinguse of the characteristic that the stroke widths of texts arenearly constant. This algorithm provides a simple way torecover the character strokes from the edge maps and can effectively extract texts in different scales and directions.

Neumann et al., [22] extracted MSERs as candidates todetect texts. After that, non-text regions are excluded by theclassifier and the reserved regions are connected into text lines.The above two methods are the two representative methods inthe field of text detection.
Chen et al., [23] proposed a novel text detection algorithm use Maximally Stable External Regions (MSER) as basic letter candidates which are filtered using geometric and stroke width data to exclude non-text objects and separate them. The character letters are paired to identify the text lines and demarcate them into words. The experimental evaluation has shown excellent performance of the proposed MSER letter candidate model.

Yao et al., [23]et al., detected a a system which detects texts of arbitrary orientations in natural images and the algorithm is equipped withtwo-level classification scheme for capturing the intrinsic characteristics of texts and then comparingthem with the state-of-the-art algorithms while handling horizontal texts and achieves significantly enhanced performance on texts of arbitrary orientations in complex natural scenes.

Neumann et al., [24] generalized a method for text localization and recognition in real world images by using hypotheses verification framework which uses various kinds of fonts to train the algorithm and then MSERs are used to detect the candidate regions for identification. The model is adaptable to the illumination changes in the scene.

Yao et al., [25] developed a model for handling multi oriented texts, where text detection and recognition are accomplished concurrently using exactly the same features and classification scheme and comparing them with a dictionary search in order to correct the recognition errors. A novel image database with texts of different scales, colors, fonts, and orientations in diverse realworld scenarios was generated and released for general use.

\section{VI.CONCLUSION}

The survey gives a very deep and broad idea about the various techniques and methods used in detection of traffic signs. This urges the curiosity and interest in the viewer to enter into the domain of traffic sign detection in real time using a better model to overcome the various problems outlined by the different methods above. Further, the future is dependent on such driver assistance systems by various governments across the world to enable a safer and secure travel.

\section{REFERENCES}

[1] R. Timofte, K. Zimmermann, and L. Van Gool, "Multi-view traffic signdetection, recognition, and 3D localisation," Mach. Vis. Appl., Vol. 25, No. 3, pp. 633-647, 2014

[2] A. de la Escalera, J. M. Armingol, and M. Mata, "Traffic sign recognitionand analysis for intelligent vehicles,” Image Vis. Comput., Vol. 21, No. 3,pp. 247-258, 2003

[3] J. Miura, T. Kanda, S. Nakatani, and Y. Shirai, "An active vision systemfor on-line traffic sign recognition,” IEICE Trans. Inf. Syst., Vol. E85-D, No. 11, pp. 1784-1792, 2002.

[4] A. Gonzalez, L. Bergasa, and J. Yebes, "Text detection and recognition on traffic panels from street-level imagery using visual appearance,”IEEE Trans. Intell. Transp. Syst., Vol. 15, No. 1, pp. 228-238, Feb. 2014. 
[5] Ruta, F. Porikli, S. Watanabe, and Y. Li, "In-vehicle camera trafficsign detection and recognition,” Mach. Vis. Appl., Vol. 22, No. 2.

[6] M. A. Garcia-Garrido, M. A. Sotelo, and E. Martin-Gorostiza, "Fast traffic sign detection and recognition under changing lighting conditions"

[7] N. Barnes and A. Zelinsky, "Real-time radial symmetry for speed signdetection," in Proc. IEEE Intell. Vehicles Symp.,pp. 566-571, Jun. 2004.

[8] N. Barnes, A. Zelinsky, and L. S. Fletcher, "Real-time speed signdetection using the radial symmetry detector," IEEE Trans. Intell.Transp. Syst., Vol. 9, No. 2, pp. 322-332, Jun. 2008.

[9] G. Loy and N. Barnes, "Fast shape-based road sign detection for adriver assistance system," in Proc. IEEE/RSJ Int. Conf. Intell. RobotsSyst. (IROS).,Vol. 1, pp. 70-75, Sep/Oct. 2004.

[10] Kwangyong Lim,1 Yongwon Hong,1 Yeongwoo Choi,2 and Hyeran Byun1, ${ }^{*}$ I. M. Creusen, R. G. J. Wijnhoven, E. Herbschleb, and P. H. N. de With,"Color exploitation in hog-based traffic sign detection," in Proc. $17^{\text {th }}$ IEEE Int. Conf. Image Process. (ICIP), pp. 2669-2672, Sep. 2010.

[11] X. Baró, S. Escalera, J. Vitrià, O. Pujol, and P. Radeva, "Trafficsign recognition usingevolutionary adaboost detection and forestECOC classification," IEEE Trans. Intell. Transp. Syst., Vol. 10, No. 1,pp. 113-126, Mar. 2009.

[12] Accurate and Efficient Traffic Sign Detection Using Discriminative AdaBoost and Support Vector Regression Tao Chen and Shijian Lu

[13] A Frequency-Domain Implementation of a Sliding-Window Traffic Sign Detector for Large Scale Panoramic DatasetsIvoCreusen

[14] Traffic Sign Detection Using a Cascade Method With Fast Feature Extraction and Saliency TestDongdong Wang ; XinwenHou ; JiaweiXu ; ShigangYue ; Cheng-Lin Liu IEEE Transactions on Intelligent Transportation Systems, Vol. 18, No. 12 , Dec. 2017.

[15] B. Epshtein, E. Ofek, and Y. Wexler, "Detecting text in natural sceneswith stroke widthtransform,” in Proc. CVPR., pp. 2963-2970, 2010.
[16] H. Chen, S. S. Tsai, G. Schroth, D. M. Chen, R. Grzeszczuk, andB Girod, "Robust text detection in natural images with edgeenhancedmaximally stable extremal regions," in Proc. ICIP., pp. 2609-2612, 2011.

[17] C. Yao, X. Bai, W. Liu, Y. Ma, and Z. Tu, "Detecting texts of arbitraryorientations in natural images," in Proc. CVPR., pp. 10831090, 2012.

[18] L. Neumann and J. Matas, "Real-time scene text localization andrecognition,” in Proc. CVPR., pp. 3538-3545, Jun. 2012.

[19] C. Yao, X. Bai, and W. Liu, "A unified framework for multiorientedtextdetection and recognition," IEEE Trans. Image Process., Vol. 23, No. 11,pp. 4737-4749, Nov. 2014.

[20] X.-C. Yin, X. Yin, K. Huang, and H.-W. Hao, "Robust text detection innatural scene images," IEEE Trans. Pattern Anal. Mach. Intell., Vol. 36, No. 5, pp. 970-983, May 2014

[21] Y.-F. Pan, X. Hou, and C.-L. Liu, "A hybrid approach to detect andlocalize texts in natural scene images," IEEE Trans. Image Process., Vol. 20, No. 3, pp. 800-813, Mar. 2011.

[22] Z. Zhang, C. Zhang, W. Shen, C. Yao, W. Liu, and X. Bai, "Multioriented text detection with fully convolutional networks," in Proc.CVPR., pp. 4159-4167, 2016.

[23] C. Yao, X. Bai, N. Sang, X. Zhou, S. Zhou, and Z. Cao,"Scene text detection via holistic, multi-channel prediction,” CoRR., 2016.

[24] J. Long, E. Shelhamer, and T. Darrell, "Fully convolutional networksfor semantic segmentation,” in Proc. CVPR., pp. 34313440, 2015.

[25] R. Girshick, “Fast R-CNN,” in Proc. ICCV, 2015, pp. 1440-1448M. Liao, B. Shi, X. Bai, X. Wang, and W. Liu, "TextBoxes: A fast textdetector with a single deep neural network," in Proc. 31st AAAI Conf.Artif. Intell. (AAAI).,pp. 4161-4167, 2017.

[26] Z. Zhong, L. Jin, S. Zhang, and Z. Feng, "DeepText: A unifiedframework for text proposal generation and text detection in naturalimages,”[Online].Available:https://arxiv.org/abs/1605.07314,2 016. 